\title{
UTILIZAÇÃO DA SIMULAÇÃO DISCRETA NA GESTÃO DE ESTOQUES DE SOBRESSALENTES PARA TURBOMÁQUINAS
}

\author{
Lidiane Borges Silverio \\ Universidade Federal Fluminense, Instituto de Ciência e Tecnologia \\ lidi.silverio@gmail.com \\ Edwin Benito Mitacc Meza \\ Universidade Federal Fluminense, Instituto de Ciência e Tecnologia \\ emitacc@id.uff.br \\ Dalessandro Soares Vianna \\ Universidade Federal Fluminense, Instituto de Ciência e Tecnologia \\ dalessandrosoares@yahoo.com.br \\ Iara Tammela \\ Universidade Federal Fluminense, Instituto de Ciência e Tecnologia \\ iaratammela@gmail.com \\ Máximo Concepción Mitacc Meza \\ Universidad de Lima, Facultad de Ingeniería Industrial \\ mmitacc@correo.ulima.edu.pe
}

\section{Resumo}

A competitividade vem aumentando em todos os segmentos da indústria. No Brasil, as indústrias do setor de petróleo têm recentemente se deparado com o grande desafio de viabilizar a produção em um ambiente de maior complexidade de acesso e restrições operacionais, dentro de um cenário de cortes de orçamento e controle de custos. Sendo assim, a cadeia de suprimentos tem importante papel para a otimização de processos, na redução de custos de aquisição e estocagem. Neste contexto, o presente artigo trata da gestão de estoques de peças sobressalentes de uma oficina de manutenção de turbomáquinas de uma empresa petrolífera. A gestão de estoques de manutenção possui grande complexidade, devido as características intermitente e errática da demanda. Propõe-se então, neste trabalho, a utilização de técnicas de simulação discreta para comparação de diferentes políticas de gestão de estoques. Espera-se conhecer melhor os impactos das alterações nos níveis e políticas de estoques em toda a cadeia de suprimentos e estoques, visando adequar os níveis de estoque de sobressalentes aos níveis de serviço requeridos e otimizar os recursos imobilizados em estoque.

Palavras-Chaves: Estoques; Manutenção; Simulação Discreta.

\begin{abstract}
The competitivity has increasing in all industry segments. In Brazil, oil and gas industries have recently faced the challenge of making feasible the production in complexity settings with operational constraints, budget cuts and cost control. Thus, the supply chain plays an important role to optimize processes and reduce acquisition and storage costs. In this context, this article deals with the inventory management of spare parts of a turbomachinery maintenance shop for an oil company. The management of maintenance inventory is very complex, due to intermittent and erratic characteristics of demand. The purpose of this paper is to use discrete simulation techniques for comparing different inventory management policies. The results of this paper help to know the impacts of changes in parameters and inventory policies, in order to balance the inventory parameters and the service levels required, leading to optimize the capital invested in inventory.
\end{abstract}

Keywords: Inventory; Maintenance; Discrete Event Simulation. 


\section{INTRODUÇÃO}

Nos dias de hoje, a indústria do petróleo mundial é reconhecida por seus grandes investimentos em pesquisa e desenvolvimento e pela relevância nos cenários políticos, sociais e econômicos de onde está inserida. No Brasil não é diferente, a indústria de óleo e gás tem papel fundamental no desenvolvimento econômico e social do país, particularmente na geração de emprego e renda, no desenvolvimento de novas tecnologias e disseminação de conhecimento.

Assim, o grande desafio para o desenvolvimento da indústria petrolífera brasileira é viabilizar a produção em águas profundas, à grandes distâncias da costa, o que demanda maiores esforços logísticos e de planejamento. Neste ambiente adverso, a manutenção tem sua importância aumentada em função da dificuldade de acessibilidade, das restrições operacionais e da preocupação constante com segurança e meio-ambiente.

Nas últimas décadas, houve uma grande evolução do conceito de manutenção em todo o mundo, o que também contribuiu para tornar esta função vital para a competitividade e sobrevivência das empresas que atuam no setor de óleo e gás. Os conceitos de gestão da manutenção e controle de custos também se desenvolveram ao longo dos últimos anos.

É dentro deste contexto que se insere o objeto deste estudo, a Oficina de Manutenção de Turbomáquinas de uma empresa do setor de óleo e gás. A oficina é uma instalação em terra que realiza a manutenção de equipamentos dinâmicos responsáveis por comprimir o gás produzido para transporte e gerar a energia necessária à produção das plataformas off-shore.

Sabe-se que um equipamento de médio porte revisado pela oficina de manutenção de turbomáquinas pode ser composto de até 1000 itens, passíveis de reposição, itens estes que podem assumir custos elevados, considerando-se que fazem uso de super ligas para suportar as altas temperaturas de operação. A demanda para estes itens ocorre em intervalos irregulares e pouco frequentes. Soma-se ao problema o fato do mercado de turbomáquinas ser pouco competitivo, geralmente é o próprio fabricante que comercializa os sobressalentes, que são importados. Desta forma, os prazos de entrega são longos, as entregas sofrem atrasos e as flutuações de preços são grandes.

Diante deste cenário complexo, a gestão de estoques de sobressalentes de turbomáquinas se torna fundamental para a redução de custos operacionais da companhia e garantia da continuidade operacional dos poços produtores de petróleo.

Segundo Altay e Litteral (2011), o gerenciamento de sobressalentes é complexo e muito difícil. De acordo com os autores, uma falha custa normalmente entre 100 a 10000 vezes o preço dos sobressalentes ou serviços envolvidos, no entanto, manter em estoque o que não é necessário gera custos e não contribui para uma cadeia de suprimentos efetiva, eficiente e responsiva.

Os itens de estoque de manutenção geralmente possuem demanda intermitente (SYNTETOS; BOYLAN, 2011), e errática (BARTEZZAGHI; KALCHSCHMIDT, 2011), o que torna mais difícil a proposição de um modelo analítico.

É por este motivo que este estudo propõe a aplicação de um modelo de simulação discreta para definição da melhor política de estoques de uma oficina de manutenção de turbomáquinas, considerando-se medidas de desempenho pré-definidas. Pretende-se comparar as políticas de reposição contínua e periódica de estoques para itens de criticidade elevada. Estes itens terão seu desempenho comparado no que diz respeito aos custos de estoque e nível de serviço.

\section{A GESTÃO DE ESTOQUES}

A gestão de estoques tem um papel importante para a competitividade das empresas, visto que deve equilibrar os custos do capital imobilizado e os custos da falta do item. De acordo com Chopra e Meindl (2007), os estoques existem na cadeia de suprimento devido a 
incompatibilidade entre fornecimento e demanda. Ainda, segundo os autores, os estoques têm um impacto significativo no tempo de fluxo dos materiais dentro da cadeia de suprimentos.

A despeito da crescente utilização de políticas de Just in Time, Gaither e Frazier (1999) citam os motivos para se manter estoque: Redução de Custos de Emissão de Pedidos, Redução de custos de stockout e redução de custos de aquisição para lotes maiores.

Garcia et al. (2006), afirmam que as principais decisões de estoque são Quanto Pedir, Quando Pedir e com que Frequência revisar os parâmetros de estoque. São estas particularidades que diferenciam os vários modelos e políticas de estoque existentes. Segundo Santoro e Freire (2008), os primeiros modelos de gestão de estoques disponibilizados durante a $2^{\text {a }}$ Guerra Mundial são denominados reativos, pois permitem tomar as decisões de quando e quanto abastecer sem que seja necessário obter previsões sobre a demanda. Mais recentemente, a abordagem ativa de reposição de estoques foi muito discutida. Essa abordagem utiliza a previsão de demanda para ajuste de parâmetros de estoque.

Ainda de acordo com Santoro e Freire (2008), o aumento da complexidade dos problemas surgidos com os modelos ativos favoreceu a utilização de simulação e de modelos de busca como instrumentos de tomada de decisões. Rosa et al. (2010) também afirmam que, com o desenvolvimento e aprimoramento do controle de estoques, os decisores tem-se utilizado de outras ferramentas como a simulação para a identificação de estratégias ótimas de gestão de estoques. A seguir serão apresentados os principais modelos de reposição de estoques.

\subsection{LOTE ECONÔMICO DE COMPRA (LEC)}

O modelo de lote econômico de compra foi o primeiro método de controle de estoques publicado por Whitman Harris em 1913, se tornando alvo de inúmeras pesquisas que originaram novos modelos. Segundo Rosa et al. (2010), o LEC obtém a quantidade ótima a ser solicitada como função dos custos de manutenção do estoque e de realização de um pedido. É um método que foi bastante criticado por tender a aumentar níveis de estoque, visto que minimiza os custos de pedido e manutenção dos estoques. Este LEC é calculado usando a seguinte equação:

$$
Q^{*}=\sqrt{\frac{2 K D}{i C}}
$$

Onde, $\mathrm{Q}^{*}$ é a quantidade ótima de pedido, $K$ é o custo fixo do pedido, $D$ é a demanda, $i$ é igual a taxa de encargos sobre o estoque e C é o custo unitário do produto.

\subsection{POLÍTICA DE REPOSIÇÃO CONTÍNUA}

A política de reposição contínua consiste no monitoramento contínuo dos estoques, de forma que a cada movimentação o saldo seja verificado para determinar se uma nova compra será necessária. De acordo com Tubino (2007), o modelo consiste em estabelecer um nível fixo de reposição $(r)$ ou ponto de pedido $(P P)$ que, ao ser atingido, dispara a emissão de um novo pedido de tamanho $Q$ pré-definido. O nível fixo de reposição $r$ é dado pela equação (2), onde $d$ é a demanda por unidade de tempo, o tempo $t$ é o tempo de reposição e $Q_{s}$ é o estoque de segurança:

$$
r=d . t+Q_{s}
$$

O estoque de segurança $Q_{s}$ é calculado usando a equação (3), multiplicando-se $m$, o número de desvios padrão para o nível de serviço especificado pelo desvio padrão da demanda durante o tempo de ressuprimento.

$$
Q s=m \sigma
$$




\subsection{POLÍTICA DE REPOSIÇÃO PERIÓDICA}

De acordo com Gaither e Frazier (1999), o modelo de gestão periódica revisa os níveis de estoque em intervalos de tempo fixos, e são feitos pedidos de material suficiente para devolver os níveis de estoque a um certo nível pré-determinado. A quantidade a ser reposta poderá variar de acordo com a demanda e níveis de estoque em cada revisão. A quantidade a ser reposta deve ser suficiente para garantir a demanda até a próxima revisão considerando o tempo de ressuprimento (TUBINO, 2007). A fórmula de cálculo da quantidade Q é dada por:

$$
Q=d \cdot\left(t_{r}+t\right)-Q_{f}-Q_{p}+Q_{r}+Q_{s}
$$

$Q_{f}$ é o saldo restante em estoque no momento da revisão. $Q_{p}$ diz respeito a quantidades em pedido em revisões anteriores que ainda não entraram em estoque, esta situação ocorre quando o tempo entre revisões é menor do que o tempo de ressuprimento. $Q_{r}$ representa quantidades solicitadas em estoque que não foram atendidas.

O tempo ótimo entre as revisões $t_{r}$ pode ser calculado utilizando a metodologia de lote econômico de compra, como proposto por Tubino (2007), onde $t_{\text {ano }}$ é igual ao número de dias do ano e $D$ é a demanda do item para o período

$$
t_{r} *=\frac{Q^{*} \cdot t_{\text {ano }}}{D}
$$

Rosa et al. (2010), afirmam que a principal vantagem da reposição periódica é a flexibilidade na determinação da periodicidade a ser empregada. Já Gaither e Frazier (1999) citam como desvantagem a exigência de maiores estoques de segurança visto que este tipo de modelo tem um maior risco de rupturas de estoque.

\section{DESCRIÇÃO DO PROCESSO DE MANUTENÇÃO DE EQUIPAMENTOS}

Os equipamentos manutenidos no setor de manutenção analisado são as chamadas turbomáquinas. Estes equipamentos são rotativos de fluxo contínuo, através do qual passa um fluido que gerará energia motriz para algum equipamento acionado como é o caso das turbinas a gás, ou que receberá energia motriz do equipamento acionador e transferirá para o fluido de processo, como é o caso dos compressores centrífugos para compressão do gás produzido. De acordo com Juliani (2003), as turbomáquinas são provavelmente os componentes mais críticos em termos construtivos e para a disponibilidade operacional de qualquer planta de processo.

Na oficina de manutenção podem ser realizadas manutenções corretivas, efetuadas após a pane com o objetivo de que o equipamento retorne à operação, ou preventivas, que são feitas de acordo com critérios prescritos para evitar falhas ou degradação. A maior parte dos equipamentos manutenidos sofrem manutenção preventiva, cuja necessidade é detectada a partir de inspeções preditivas. As manutenções preventivas realizadas na oficina são chamadas de revisão geral e já tem escopo pré-definido. Os sobressalentes envolvidos neste tipo de revisão também são previamente levantados e supridos em estoque.

Será apresentado a seguir o processo de revisão geral típico de um modelo de turbina a gás, bem como o processo de reposição dos sobressalentes.

\subsection{PROCESSO DE REVISÃO DE TURBOMÁQUINAS}

As revisões (manutenções preventivas) consistem no recebimento do equipamento, desmontagem, triagem, inspeção inicial, reparo, inspeção final e montagem do equipamento. A manutenção é dividida nas etapas a seguir:

- Fase A - Desmontagem, Triagem, Cadastro, Limpeza e Inspeção inicial.

- Fase B - Reparo e Inspeção Final.

- Face C - Montagem. 
Na Fase A, o equipamento recebido é desmontado e suas peças são triadas, cadastradas e limpas (retirada de incrustações e resíduos) de modo a facilitar a próxima etapa de inspeção. Os sobressalentes envolvidos na revisão podem ser de dois tipos:

- Itens de troca obrigatória: Peças que não são inspecionados e devem ser substituídas obrigatoriamente. Esses sobressalentes são solicitados do estoque assim que a manutenção se inicia. São exemplos de itens de troca obrigatória parafusos, arruelas, gaxetas, frenos, anéis de vedação, sensores, entre outros.

- Itens passíveis de inspeção: São materiais que após a desmontagem e limpeza, passam por inspeção, que irá determinar se estão em condições de uso ou se devem ser reparados ou substituídos. Se enquadram neste tipo de item palhetas estatoras e rotoras, diafragmas, selos, etc. Este tipo de material é solicitado após o término da Fase A, quando se tem o resultado da inspeção inicial. Esses materiais têm características de demanda intermitente.

Após o término das inspeções (Fase A), os materiais que podem ser reparados seguem para reparo e os materiais a serem substituídos por novos são solicitados, podendo existir ou não em estoque. Itens de muito pouco uso normalmente não tem parâmetro de ressuprimento de estoque e são comprados sob demanda, já os de uso mais provável são mantidos em estoque. Os itens passíveis de inspeção podem ser subdivididos em dois tipos:

- Itens de Demanda unitária: Quando se utiliza apenas uma unidade do determinado item por equipamento ou quando se utiliza mais do que uma unidade do item por equipamento, mas a reprovação na inspeção inicial se dá sempre por lote, de forma que as peças são sempre substituídas em sua totalidade, podendo ser consideradas como demandas unitárias.

- Itens de Demanda errática: Quando se utiliza mais de uma unidade por equipamento e as quantidades consumidas por manutenção podem variar.

A Fase B, consiste no reparo e inspeção final, quando os materiais que retornam de reparo são inspecionados novamente. Só então os materiais solicitados no início da Fase A, no caso dos itens de troca obrigatória e ao término da Fase A para os casos de itens inspecionáveis, precisam estar disponíveis para a fase final de montagem. A partir deste ponto a falta de sobressalentes afeta o andamento da revisão do equipamento (Figura 1). Caso a oficina esteja com sua capacidade de manutenção de equipamentos comprometida quando da chegada de um novo equipamento para revisão, pode-se optar por enviá-lo para outra oficina reparadora.

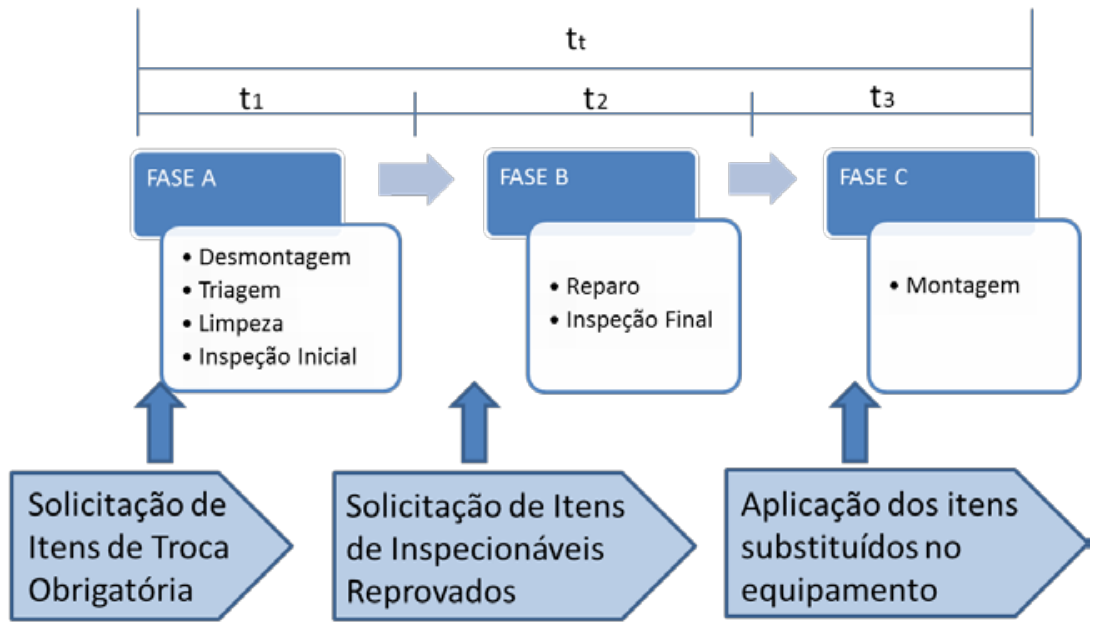

Figura 1. Solicitação e aplicação de sobressalentes durante manutenção. Fonte: Própria. 


\subsection{PROCESSO DE REPOSIÇÃO DE SOBRESSALENTES}

Quando é feita uma solicitação de material durante a manutenção, o sistema de planejamento de materiais (MRP - Materials Resource Planing), implantado na empresa, irá comparar os parâmetros de estoque cadastrados para o material com o saldo em estoque e as necessidades colocadas, gerando assim uma requisição de compra, caso necessário.

A requisição de compra, logo que é gerada, deve ser analisada por uma equipe responsável, que irá encaminhar para o grupo comprador daquele tipo de item. A partir daí, tem início o processo de compra, que é encerrado com a colocação e envio de pedido de compra para o fornecedor. Decorrido o prazo de fornecimento, o material é entregue e conferido para entrada em estoque. A Figura 2 ilustra o processo de reposição de materiais em estoque.

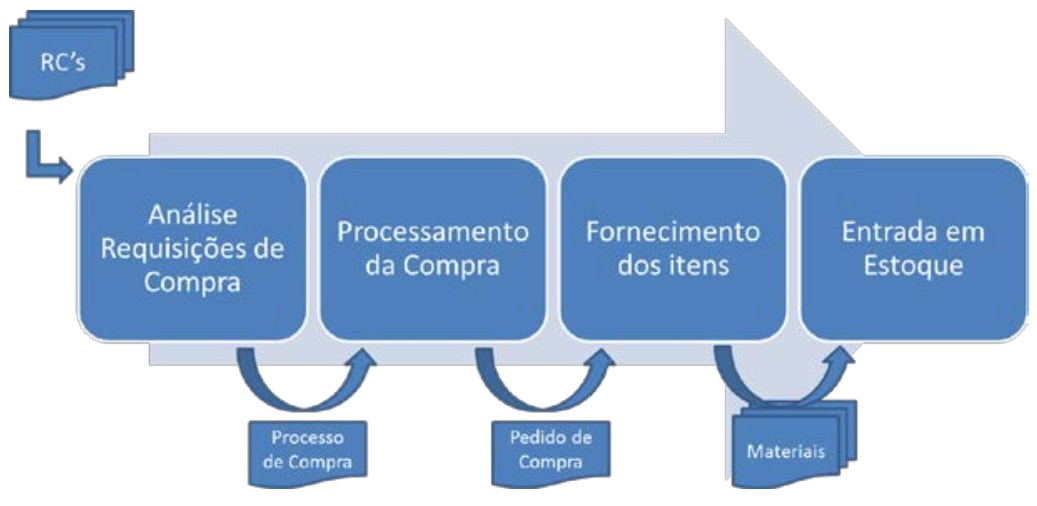

Figura 2. Processo de reposição de materiais em estoque. Fonte: Própria.

\section{ETAPAS DE UM ESTUDO DE SIMULAÇÃO}

O processo de simulação segue o método científico, ou seja, formula as hipóteses, prepara o experimento, testa as hipóteses através do experimento e valida as hipóteses através dos resultados obtidos. De acordo com Pegden (1990) apud Freitas Filho (2008), a simulação é o processo de projetar um modelo computacional de um sistema real e conduzir experimentos com o propósito de entender seu comportamento e avaliar estratégias para sua operação. Chwif \& Medina (2010) apontam alguns passos que devem ser seguidos:

1. Concepção ou formulação do modelo: Deve-se entender claramente o sistema a ser simulado e seus objetivos; Criar um modelo conceitual; Coletar os dados de entrada.

2. Implementação do modelo: Conversão do conceitual para computacional; Comparação do modelo computacional com conceitual para avaliar se a operação atende ao que foi estabelecido na etapa de concepção.

3. Análise dos resultados do modelo: Realização das rodadas do modelo; Análise e documentação dos resultados.

A seguir, serão descritos cada um destes passos para o problema de gestão de estoques de uma oficina de manutenção de turbomáquinas.

\section{CONCEPÇÃO DO MODELO}

De acordo com o processo mapeado no item 3, foram definidas as premissas para modelagem, medidas de desempenho do sistema, entidades do sistema, processos e parâmetros de entrada do modelo. Uma das premissas utilizadas foi a não inclusão no modelo dos recursos e movimentações envolvidas em cada processo, pois o objetivo deste estudo é analisar o comportamento dos estoques e seus parâmetros de ressuprimento, não sendo relevante no presente estudo o detalhe dos processos envolvidos no consumo e ressuprimento dos sobressalentes. Outra premissa utilizada foi a de restringir a modelagem para um único modelo de equipamento revisados na oficina. 
Para efeito de análise de desempenho serão verificados: Valor médio em estoque, Custo Total de Pedido, Quantidade de ocorrências de faltas de sobressalentes observadas e Tempo médio de espera do equipamento por chegada de sobressalentes.

O modelo conceitual foi construído de acordo com a Figura 3, utilizando a simbologia IDEF-SIM (LEAL et al., 2008). As entidades deste diagrama estão relacionadas na Tabela 1.

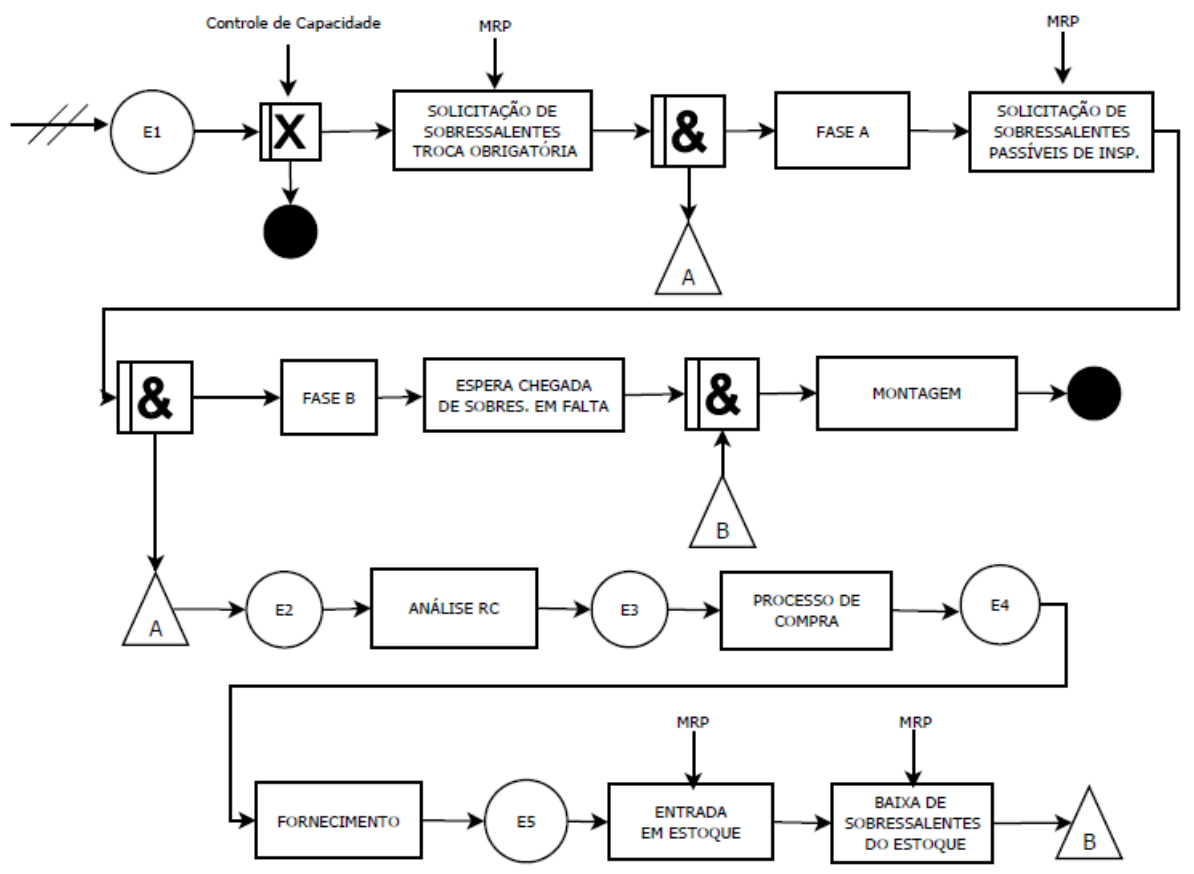

Figura 3. Modelo Conceitual linguagem IDEF-SIM. Fonte: Própria.

Tabela 1 - Entidades do Sistema.

\begin{tabular}{cl}
\hline Entidade & \multicolumn{1}{c}{ Descrição } \\
\hline E1 & Equipamento a ser revisado \\
E2 & Requisição de Compra \\
E3 & Processo de Compra \\
E4 & Pedido de Compra \\
E5 & Item sobressalente \\
\hline
\end{tabular}

É importante ressaltar que, caso a Oficina de manutenção não tenha capacidade para atendimento de um novo equipamento, ele sai do sistema. Se houver capacidade, o equipamento segue no sistema e são solicitados itens de troca obrigatória. Neste momento o controle de MRP atrelado a esta função irá gerar requisições de compra caso seja necessário, ao mesmo tempo em que será iniciada a Fase A da manutenção. Após o término da Fase A, novos sobressalentes denominados passíveis de inspeção são solicitados e o equipamento segue para a Fase $\mathrm{B}$, ao mesmo tempo em que novas requisições poderão ser geradas pelo controle de MRP. Quando a Fase B termina, caso todos os materiais solicitados não estejam disponíveis em estoque, o equipamento esperará a chegada dos itens pendentes no estoque para realizar a baixa do saldo em estoque de todos os itens necessários e executar a montagem, última fase da manutenção.

As funções de Solicitação de Sobressalentes de troca obrigatória e passíveis de inspeção poderão gerar requisições de Compra. Para os itens passíveis de inspeção, eles poderão ser ou não solicitados pelo modelo mediante histórico de utilização em revisões anteriores. As duas funções de Solicitação de Materiais possuem um controle (MRP) que consulta os parâmetros de estoque para verificar se a solicitação de materiais gerará ou não Requisição de Compra de acordo com níveis de estoque. É neste ponto do modelo que será possível introduzir as diferentes políticas e níveis de estoque para comparar o desempenho de cada uma delas. 
A função análise de Requisição de Compra irá agrupar as Requisições de cada material em um processo de compra único que será efetuado e terá como resultado a emissão do pedido de compra com os devidos prazos de fornecimento de cada item. Após o fornecimento, a entidade analisada passa a ser o item sobressalente entregue pelo fornecedor, que levará um tempo para processamento e entrada no estoque, após o qual ocorrerá a baixa de estoque, caso seja um material solicitado para algum equipamento em revisão. Após a chegada dos materiais pendentes, caso existam, a manutenção pode ser finalizada através da montagem do mesmo.

Os dados de entrada do modelo foram coletados com base em registros históricos do sistema ERP da empresa dos últimos 3 anos. Foram selecionados 10 itens sobressalentes para a simulação, nomeados de A à J. Os materiais de A à E são os de troca obrigatória e os de $\mathrm{F}$ à $\mathrm{J}$ são os do tipo passível de inspeção. A escolha destes materiais foi baseada em elevado histórico de consumo, aliado a grande criticidade dos itens para o modelo de equipamento estudado. Dos 5 materiais escolhidos do tipo passível de inspeção, os itens de $\mathrm{F}$ à $\mathrm{H}$ possuem comportamento de demanda unitária e os materiais I e J possuem demanda errática. Para análise dos dados foi utilizado o pacote ExpertFit do software de Simulação FlexSim.

A Tabela 2 apresenta o percentual histórico de utilização para os itens de demanda unitária. Já a Tabela 3 apresenta a unidade de medida utilizada e a distribuição de probabilidade aplicada para cada dado de entrada no modelo. É importante ressaltar que o tempo de processamento não se ajustou a nenhuma distribuição de probabilidade e foi utilizada então uma distribuição empírica. O tempo entre chegada de equipamentos foi o único parâmetro que teve dados históricos coletados de um prazo maior que 3 anos, foram utilizados 10 anos para melhorar a previsão de demanda de manutenção de equipamentos. Ressalta-se que durante o período de 10 anos não houve alterações da frota em operação deste modelo de equipamento.

\begin{tabular}{|c|c|c|}
\hline & Item & Percentual de Utilização \\
\hline & Material F & $75 \%$ \\
\hline & Material G & $68,80 \%$ \\
\hline & Material H & $56,30 \%$ \\
\hline
\end{tabular}

Tabela 3 - Distribuições de Probabilidade.

\begin{tabular}{|llll}
\hline \multicolumn{1}{c}{ Dados de entrada } & Unidade & Distribuição & \multicolumn{1}{c}{ Parâmetros Flexsim } \\
\hline Tempo entre chegadas de equipamento & Dias & Beta & beta(0.61, 405.10, 0.71, 2.15) \\
\hline Tempo de Processamento Fase A & Dias & Jonhson SB & johnsonbounded(1.35, 35.77, 0.77, 1.04) \\
\hline Tempo de Processamento Fase B & Dias & Beta & beta( 26.39, 281.81, 0.66, 1.06) \\
\hline Tempo de Processamento Análise RC & Dias & Negative Binomial & negbinomial(3.00, 17.95) \\
\hline Tempo de Processamento de Compra & Dias & Empírica & Global table \\
\hline Tempo de Proc. de Entrada de & Dias & Log-logistic & loglogistic(0.00, 2.92, 2.27) \\
Material no estoque & Dias & Jonhson SB & johnsonbounded(84.47, 260.65, -0.03, 0.70) \\
\hline Tempo de Fornecimento Material A & Dias & Log-Logistic & loglogistic(0.00, 132.96, 3.25) \\
\hline Tempo de Fornecimento Material B & Dias & Jonhson SB & johnsonbounded(34.32, 266.09, 0.18, 0.74) \\
\hline Tempo de Fornecimento Material C & Dias & Jonhson SB & johnsonbounded(51.88, 297.04, 0.27, 0.53) \\
\hline Tempo de Fornecimento Material D & Dias & Gama(E) & gamma( 38.49, 20.92, 4.59) \\
\hline Tempo de Fornecimento Material E & Dias & Inverse Gaussian & inversegaussian( 10.50, 106.75, 304.15) \\
\hline Tempo de Fornecimento Material F & Dias & Inverse Gaussian & inversegaussian( 10.50, 106.75, 304.15) \\
\hline Tempo de Fornecimento Material G & Dias & Weibull(E) & weibull( 32.75, 100.86, 1.81) \\
\hline Tempo de Fornecimento Material H & Dias & Jonhson SB & johnsonbounded(53.76, 250.70, 0.58, 0.64) \\
\hline Tempo de Fornecimento Material I & Dias & Jonhson SB & johnsonbounded(53.76, 250.70, 0.58, 0.64) \\
\hline Tempo de Fornecimento Material J & Unid. & Negative Binomial & negbinomial( 1.00, 24.17) \\
\hline Consumo I & Unid. & Negative Binomial & negbinomial( 1.00, 18.03) \\
\hline Consumo J & & & \\
\hline
\end{tabular}




\section{IMPLEMENTAÇÃO COMPUTACIONAL}

O modelo conceitual foi implementado utilizando-se o software FlexSim 7.3, Versão Acadêmica, onde todos os parâmetros de entrada e medidas de desempenho citados no item anterior foram configurados. A Figura 4 apresenta o modelo construído no software FlexSim. Para determinar o período de aquecimento do sistema foram realizadas 5 replicações e analisados os níveis de estoque e filas do sistema, chegando-se a conclusão de que o sistema entra em regime permanente com cerca de 500 dias de simulação.

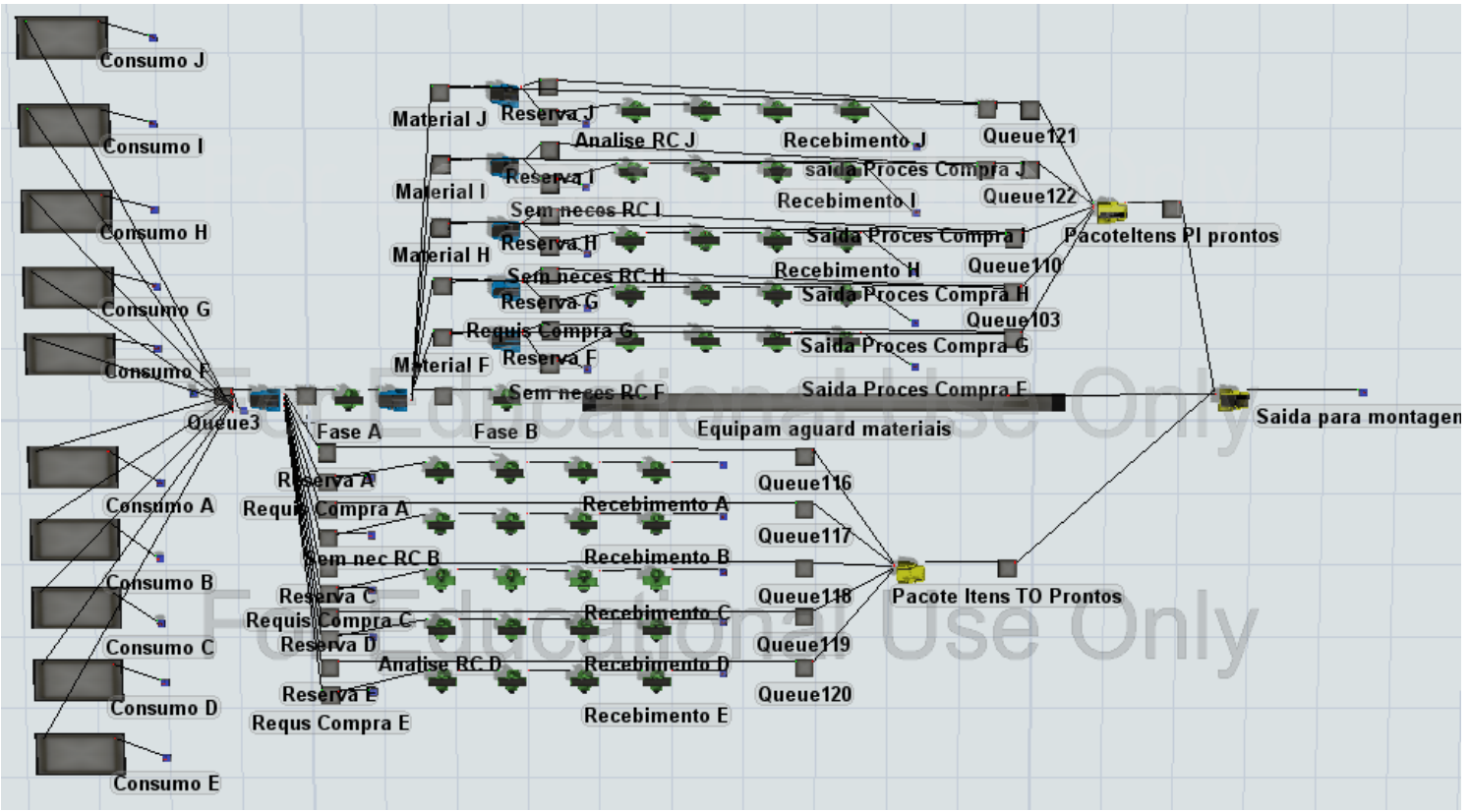

Figura 4. Modelo construído no software FlexSim.

Para encontrar o número ideal de replicações, foram realizadas 20 replicações e avaliados os resultados das medidas de desempenho para uma confiança de 95\%. De acordo com Schwif e Medina (2010), o número ideal de replicações é dado por:

$$
n^{*}=n \cdot\left(\frac{h}{h^{*}}\right)^{2}
$$

Onde $n^{*}$ é o número de replicações necessárias, $n$ é igual ao número de replicações da amostra piloto, $h$ é a precisão da amostra piloto e $h *$ é a precisão desejada.

A Tabela 4 apresenta os valores calculados de replicações mínimas para o nível de precisão desejado, onde se verifica que devem ser feitas 222 replicações. A tabela também apresenta o resultado das 222 replicações. Apesar de algumas das medidas de desempenho ficarem com precisão um pouco acima da desejada, o número de 222 replicações foi considerado satisfatório, pois os desvios em relação a precisão desejada não foram muito significativos. Foi estipulado um período de simulação de 4 anos para se aumentar a precisão do modelo, em função dos longos prazos de ressuprimento e de manutenção observados.

Para a simulação do cenário atual, cuja política de gestão de estoques é a de revisão contínua, foram incluídos no modelo os parâmetros de ressuprimento $\mathrm{R}$ e $\mathrm{R}+\mathrm{Q}^{*}$, calculados de acordo com a demanda anual para cada item e média histórica dos tempos de ressuprimento. No cenário atual os parâmetros de ressuprimento R e R+Q* são ajustados de forma que sejam divisíveis pela quantidade aplicada em cada equipamento para os casos de troca obrigatória e passíveis de inspeção com demanda unitária, evitando assim um nível de "estoque morto”, que não tem quantidade suficiente para atendimento a demanda solicitada. $\mathrm{O}$ estoque de segurança foi dimensionado para cobertura de 45 dias. Estes parâmetros estão listados na tabela 5. 
Tabela 4 - Número mínimo de replicações.

\begin{tabular}{|c|c|c|c|c|c|c|}
\hline \multirow{2}{*}{ Medida de Desempenho } & \multicolumn{4}{|c|}{$\mathrm{n}=\mathbf{2 0}$} & \multicolumn{2}{|c|}{$n=222$} \\
\hline & Média & $\mathrm{h}$ & $\mathrm{h}^{*}$ & $\mathrm{n}^{*}$ & Média & $\mathrm{h}$ \\
\hline Estoque Médio Material A & 698 & 142 & 50 & 161 & 654 & 53 \\
\hline Estoque Médio Material B & 449 & 68 & 30 & 103 & 455 & 26 \\
\hline Estoque Médio Material C & 501 & 70 & 35 & 80 & 522 & 29 \\
\hline Estoque Médio Material D & 395 & 45 & 25 & 65 & 440 & 17 \\
\hline Estoque Médio Material E & 30,5 & 5 & 2 & 125 & 29,7 & 1,8 \\
\hline Estoque Médio Material F & 121 & 19 & 8 & 113 & 119,2 & 6,3 \\
\hline Estoque Médio Material G & 64 & 17 & 5,5 & 191 & 64 & 6,7 \\
\hline Estoque Médio Material H & 2,86 & 0,42 & 0,2 & 88 & 2,661 & 0,107 \\
\hline Estoque Médio Material I & 5,12 & 0,7 & 0,35 & 80 & 5,17 & 0,47 \\
\hline Estoque Médio Material J & 10,4 & 2,8 & 0,85 & 217 & 10,07 & 0,56 \\
\hline Tempo Médio de Espera por Equipamento & 83 dias & 30 & 9 & 222 & 66,1 dias & 7,7 \\
\hline Média de Equipamentos Aguardando Materiais & 2,49 & 0,67 & 0,25 & 144 & 1,97 & 0,18 \\
\hline
\end{tabular}

Tabela 5 - Parâmetros de Ressuprimento de Estoque.

\begin{tabular}{ccccccc}
\hline & \multicolumn{4}{c}{ Revisão Contínua } & \multicolumn{2}{c}{ Revisão Periódica } \\
\cline { 2 - 7 } Material & Qs & R ajustado & $\mathrm{Q}^{*}$ & $\mathrm{R}+\mathrm{Q}^{*}$ ajustado & tr* & $\begin{array}{c}\text { d.(tr*+t) } \\
\text { ajustado }\end{array}$ \\
A & 210 & 1392 & 360 & 1856 & 77 & 1646 \\
B & 114 & 696 & 474 & 1160 & 186 & 1278 \\
C & 91 & 588 & 714 & 1176 & 354 & 1477 \\
D & 62 & 378 & 652 & 1008 & 476 & 1072 \\
E & 8 & 51 & 5 & 68 & 30 & 43 \\
F & 29 & 176 & 5 & 264 & 8 & 235 \\
G & 22 & 82 & 5 & 164 & 10 & 142 \\
H & 0 & 3 & 1 & 4 & 183 & 2 \\
I & 1 & 6 & 1 & 7 & 46 & 6 \\
J & 2 & 13 & 2 & 15 & 43 & 12 \\
\hline
\end{tabular}

Os resultados para o cenário atual encontram-se na Tabela 6. É importante ressaltar que após a execução do modelo computacional, que busca de maneira simplificada retratar o cenário atual, foram comparados os resultados desta simulação com os dados levantados pela coleta realizada. Com base nos dados gerados como resultado do modelo de simulação foi possível verificar a aderência destes com a realidade.

Tabela 6 - Medidas de Desempenho Cenário Atual.

\begin{tabular}{lr}
\hline \multicolumn{1}{c}{ Medida de Desempenho } & \multicolumn{1}{c}{ Valor } \\
\hline Valor Médio em Estoque & $\mathrm{R} \$ 2.593 .319,20$ \\
\hline Custo Total de Pedidos & $\mathrm{R} \$ 56.270,44$ \\
Tempo médio de Espera por Equipamento & 66,1 dias \\
Número Médio de Ocorrência de Faltas de Itens & 63,84 \\
\hline
\end{tabular}

\section{PROPOSTAS DE CENÁRIOS ALTERNATIVOS}

Foram simulados cenários alternativos com o objetivo de comparar com o cenário atual, buscando melhores resultados, que equilibrem o valor imobilizado em estoque com o nível de serviço desejado. Para tanto, foram escolhidos dois cenários alternativos, o primeiro utilizando-se de política de revisão periódica de parâmetros de estoque (Cenário Alternativo 1) e o segundo analisando o impacto de se estabelecer contrato de longo prazo com o fornecedor (Cenário Alternativo 2). O contrato de longo prazo diminui o tempo de processamento da 
compra, apesar de não alterar o prazo de fornecimento, haja vista que o fornecedor mantem os mesmos prazos de fornecimento para compras com e sem contrato.

Foram realizados reajustes nos dados de entrada do modelo para os dois cenários alternativos. Os parâmetros de ressuprimento utilizados para o cenário alternativo 1 estão listados na Tabela 5. Para o cenário alternativo 2, foram coletados dados históricos de compras realizadas através de contrato e encontrada a seguinte distribuição de probabilidade para o tempo de processamento da compra: Exponential(0.00, 5.10).

Através das Figuras 5 e 6 é possível comparar o resultado das principais medidas de desempenho para cada cenário. Percebe-se que no cenário alternativo 1, os níveis de serviço permanecem praticamente iguais aos do cenário atual, porém o valor médio em estoque teve um aumento de $8 \%$ e os custos de pedido também sofreram aumento. Já o cenário alternativo 2 apresentou uma queda de $23 \%$ nos estoques médios, acompanhada de pequena alta nos custos de pedido, além de apresentar melhores resultados no Tempo Médio de Espera.

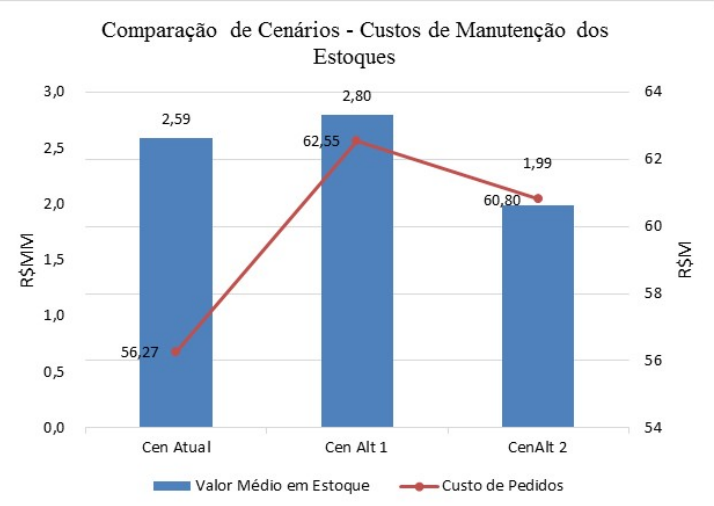

Figura 5. Custos de Estoques

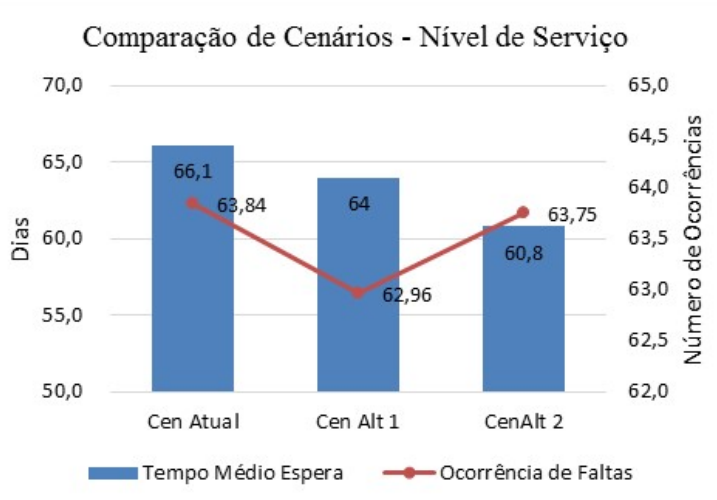

Figura 6. Nível de Serviço

A Figura 7 estratifica as ocorrências de falta de material por item. Percebe-se que os números de ocorrências de faltas para cada item são muito próximos uns dos outros para todos os cenários simulados. Os itens I e J são os que mais tiveram ocorrência de falta nos 3 diferentes cenários, devido ao caráter aleatório de consumo, cuja característica da demanda é errática. Sendo assim, foi realizada uma última simulação utilizando-se os parâmetros do cenário atual, mas aumentando-se os parâmetros apenas dos itens I e J, de acordo com a média do terceiro quartil de consumo destes itens registrados nas simulações anteriores. Os resultados da Tabela 7, mostram uma melhora nos níveis de serviço do modelo. Com esta pequena alteração, o tempo médio de espera de equipamento caiu de 66,1 dias para 56,1 dias, uma redução de $18 \%$ com incremento nos estoques de apenas 1,7\%.

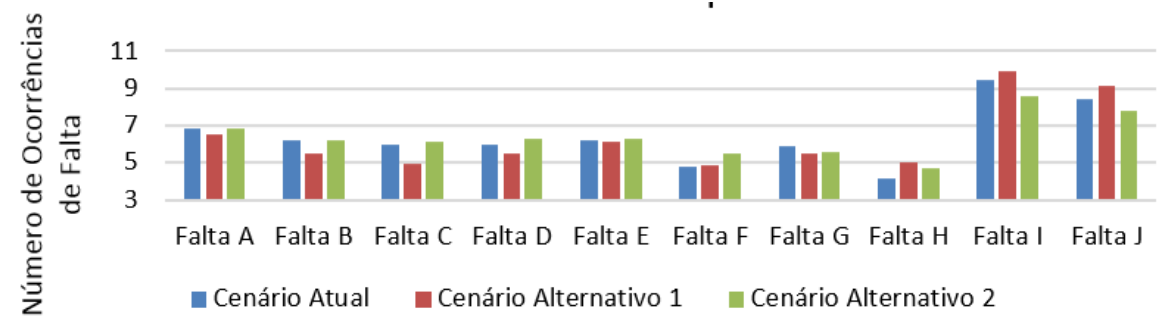

Figura 7. Ocorrência de Faltas por item.

Tabela 7-Medidas de Desempenho Cenário Atual com parâmetros de I e J ajustados.

\begin{tabular}{lr}
\hline \multicolumn{1}{c}{ Medida de Desempenho } & \multicolumn{1}{c}{ Valor } \\
\hline Valor Médio em Estoque & $\mathrm{R} \$ 2.637 .828,17$ \\
\hline Custo Total de Pedidos & $\mathrm{R} \$ 56.715,68$ \\
Tempo médio de Espera por Equipamento & 56,1 dias \\
Número Médio de Ocorrência de Faltas de Itens & 63,32 \\
\hline
\end{tabular}




\section{CONCLUSÕES}

Com base nos resultados obtidos nas simulações dos três diferentes cenários, concluise que o modelo de revisão periódica é o menos eficiente para o problema em questão, pois apresentou níveis de estoque mais elevados para o mesmo nível de serviço do modelo de revisão contínua. Este fato corrobora a afirmação de Gaither e Frazier (1999), de que este tipo de modelo exige maiores níveis de estoque devido ao elevado risco de ruptura de estoque. Verificou-se também que o cenário alternativo 2, de revisão contínua com a utilização de um contrato, pode trazer grandes economias de estoque, mantendo-se o mesmo nível de serviço. Além disto, constata-se que o ajuste seletivo de parâmetros nos itens com maior ocorrência de faltas pode aumentar os níveis de serviço, com pequenos incrementos no estoque.

O modelo se mostrou satisfatório para auxiliar nas tomadas de decisões referentes a políticas de gestão de estoques, pois tornou possível quantificar os impactos de alterações em parâmetros de ressuprimento e tipos de políticas de estoque, bem como avaliar novas estratégias e ações a serem implementadas para otimização dos processos da cadeia de suprimento.

\section{REFERÊNCIAS BIBLIOGRÁFICAS}

[1] ALTAY, N.; LITERAL, L. (Edit). Services Parts Management: Demand Forecasting and Inventoy Control, $3^{\mathrm{a}}$ ed. Springer: London, 2011.

[2] BARTEZZAGHI, E.; KALCHSCHMIDT, M. The impact of agregation level on lumpy demand management. In: ALTAY, N.; LITERAL, L. (Edit). Services Parts Management: Demand Forecasting and Inventoy Control, $3^{\mathrm{a}}$ ed. Springer: London, 2011.

[3] CHOPRA, S.; MEINDL, P. Supply chain management: Strategy, Planning and Operation. New Jersey: Prentice Hall, 2007.

[4] CHWIF, L.; MEDINA, A. Modelagem e Simulação de Eventos Discretos: Teoria e Aplicações, $3^{\mathrm{a}}$ edição. Rio de Janeiro: Elsevier, 2010.

[5] FREITAS F. Introdução a Modelagem e Simulação de Sistemas com Aplicações em Arena, $2^{\mathrm{a}}$ edição. São Paulo: VisualBook, 2008.

[6] GAITHER, N.; FRAZIER, G. Administração da Produção e Operações 8 a ed. São Paulo: Pioneira Thomson Learnig, 1999

[7] GARCIA, E.; REIS, L.; MACHADO, L. \& FERREIRA FILHO, V.J. Gestão de estoques: otimizando a logística e a cadeia de suprimentos. Rio de Janeiro: E-papers, 2006.

[8] JULIANI, A. Turbomáquinas Principais Modelos e Tipos Usados Em Plantas De Separação De Gases - Teoria E Prática. In: XVIII Encontro de Produtores e Consumidores de Gases Industriais, 2003, São Paulo.

[9] LEAL, F.; ALMEIDA, D. A.; MONTEVECHI, J. A. B. Uma proposta de técnica de modelagem conceitual para a simulação através de elementos do IDEF. In: Simpósio Brasileiro de Pesquisa Operacional, 2008. João Pessoa-PB.

[10] ROSA, H.; MAYERLE, S.; GONÇALVES, M. Controle de estoque por revisão contínua e revisão periódica. Produção, v. 20, n. 4, dez. 2010.

[11] SANTORO, C. M.; FREIRE, G. Análise comparativa entre modelos de estoque. Revista Produção, v. 18, n. 1, p. 89-98, 2008.

[12] SYNTETOS, A.; BOYLAN, J. Intermittent demand: Estimation and Statistical Properties, In: ALTAY, N.; LITERAL, L. (Edit). Services Parts Management: Demand Forecasting and Inventoy Control, $3^{\mathrm{a}}$ ed. Springer: London, 2011.

[13] TUBINO, D. F. Planejamento e Controle da Produção. $1^{\text {a }}$ ed. Editora Atlas, 2007. 